\section{Präventive INH-Therapie bei der Behandlung von Atemwegs- und Lungenerkrankungen mit Kortikosteroiden?}

Unter präventiver Chemotherapie versteht man die Anwendung von Antituberkulotika bei tuberkulinpositiven Personen; Chemoprophylaxe beinhaltet die Therapie von Personen mit negativem Tuberkulintest zur Verhütung der Infektion bei Gefährdung (Exposition bzw. Immunsuppression). Diese Unterscheidung wird nicht immer streng eingehalten, sie erleichtert jedoch die therapeutische Entscheidung. Es besteht kein Zweifel daran, dass die Therapie mit systemischen Kortikosteroiden das Risiko, eine aktive Tuberkulose zu induzieren, grundsätzlich erhöhen [1]. Die wichtigsten pneumologischen Erkrankungen, die mit Kortikosteroiden, teils auch über längere Zeit behandelt werden, sind das Asthma bronchiale, die chronisch obstruktive Atemwegserkrankungen mit und ohne Emphysem sowie interstitielle Lungenerkrankungen, hier insbesondere auch die Sarkoidose.

Bis ca. 1975 gab es in Deutschland wenig Zweifel an der Sinnhaftigkeit einer INH-Therapie bei notwendiger Kortikosteroid-Langzeittherapie pneumologischer Erkrankungen, aber auch anderer chronischer Erkrankungen wie Rheumatoider-Arthritis, speziellen Anämieformen usw. Allerdings war die Literatur zu diesem Thema auch damals sparsam $[5,4,2,1,3,6]$. Beschrieben werden sowohl reaktivierte Tuberkulosen als auch neu aufgetretene Tuberkulosen bei Tuberkulin-positiven Patienten. Die in aller Regel durchgeführte INH-Monotherapie war bei ausgeprägter Immunsuppression jedoch gelegentlich nicht in der Lage, eine aktive Tuberkulose zu verhindern, wie R. Kropp an 4 außergewöhnlichen Fällen beobachten konnte [7]. Die Etablierung der modernen antituberkulotischen Chemotherapie führte naturgemäß zu einem deutlichen Rückgang der Tuberkulosehäufigkeit und dadurch auch zu einem Rückgang prophylaktischer Behandlungen.

Empfohlen wird die präventive Chemotherapie auch im neuen Tuberkulosebuch [8] im Kapitel Therapie der Tuberkulose von G. Siemon und R. Kropp, Seite 136 und zwar wird als Indikation eine längerfristige Kortikosteroidbehandlung in „überphysiologischer“ Dosis genannt. Die aktuellen Empfehlungen des ATS von 2000 [9] zeigen das relative Risiko zur Entwicklung einer aktiven Tuberkulose bei wenigen klinischen Bedingungen an (siehe Tab.1). Anzumerken ist jedoch, dass eine Erhöhung des relativen Risikos nicht in jedem Fall eine prophylaktische oder präventive Chemotherapie bedingt! Ich

Pneumologie 2000; 54: 419-420

(C) Georg Thieme Verlag Stuttgart · New York ISSN 0934-8387

\section{Kirsten}

Zentrum for Pneumologie und Thoraxchirurgie, Großhansdorf
Tab. 1 Relative Risiko für die Entwicklung einer aktiven Tuberkulose klinische Bedingungen relatives Risiko (unabhängig vom Tuberkulinstatus)

\begin{tabular}{ll}
\hline Silikose & 30 \\
Diabetes mellitus & $2-4$ \\
chronische Nierenkrankheiten & $10-25$ \\
Z. n. Gastrektomie & $2-5$ \\
Organtransplantation - Niere & $20-70$ \\
& \\
- Herz &
\end{tabular}

Tab. 2 Maßnahmen vor Beginn einer Prävention (Chemotherapie/ Chemoprophylaxe) mit INH

Tuberkulintestung

aktive Tuberkulose ausschließen

anamnestisch vorausgegangene antituberkulotische Therapie ausschließen

nach vorausgegangener Hepatopathie fahnden, ggf. Hepatitisserolgie durchführen

INH nur für maximal 4 Wochen rezeptieren (Compliance!)

kann mich in den letzten 20 Jahren nur auf 2-3 Patienten besinnen, bei denen ein Kausalzusammenhang zwischen der Kortison-Langzeittherapie bei Patienten mit Asthma bronchiale und der Tuberkuloseentwicklung für möglich gehalten wurde. Bei der Kortisontherapie der Sarkoidose ist mir ein derartiger Zusammenhang bisher nicht begegnet! Allerdings habe ich das gleichzeitige Auftreten beider Erkrankungen in einigen Fällen beobachtet. Hier wurde dann eine regelrechte antituberkulotische Therapie parallel zu einer Kortisontherapie erfolgreich durchgeführt. In letzter Zeit wurde aus Mexiko eine Fallkontrollstudie aus den Jahren 1987 bis 1992 publiziert [10]. Die Autoren folgern aus ihrer Studie, dass ein Nutzen für eine INH-Prophylaxe nur in Ländern mit hoher Tuberkuloseprävalenz besteht. Diese Studie schloss jedoch nur Patienten mit einer chronischen Rheumatoider Arthritis und Kortison-Langzeittherapie ein. In einem Review zum Thema Kortikosteroide und Tuberkulose kommen Senderovitz und Viskum [11] zum Schluss, dass aus neueren prospektiven randomisierten, plazebokontrollierten Studien kein sicherer Nutzen für eine präventive Therapie bei Patienten mit positivem Tuberkulintest bzw. früher durchgemachter Tuberkulose durch eine INH-Monotherapie zu sehen ist. Generell sind 
die Nebenwirkungen der langmonatigen INH-Therapie gegen den potenziellen Nutzen immer wieder zu bedenken. Die durchzuführenden Maßnahmen vor Beginn einer präventiven Chemotherapie/Chemoprophylaxe mit INH, wie sie von Bartmann bereits 1973 postuliert wurden, sind weiterhin aktuell und sinnvoll ([12], Tab. 2).

Die eigenen Erfahrungen und die Analyse der Literaturdaten führen mich zu dem Schluss, dass die Durchführung einer präventiven Chemotherapie bzw. Chemoprophylaxe mit INH bei Kortikosteroid-Langzeittherapien bei Atemwegs- und Lungenkrankheiten nicht generell zu empfehlen ist. Nutzen und leberbezogenes Risiko stehen für mich in keinem vertretbaren Verhältnis. Ich rate deshalb zu einer konsequenten klinischen Beobachtung mit regelmäßiger Überprüfung des klinischen Zustandes (Anamnese, Untersuchungsbefunde) sowie radiologischen Kontrollen in ca. 4- bis 6-monatigem Abstand. Für die gelegentlich geäußerte Meinung, dass eine Tagesdosis $>15 \mathrm{mg}$ Kortison das Tuberkuloserisiko steigert, gibt es keine Evidenz. Bei höheren Dosen sollten die klinischen Kontrollen besonders konsequent durchgeführt werden, und wenn der klinische oder radiologische Verdacht auf das Vorliegen einer Tuberkulose besteht, eine regelrechte Therapie mit mindestens 3 Antituberkulotika erfolgen. Diese Meinung wird unterstützt durch die Tatsache, dass sogar bei durch retrovirale Infektion immunkompromittierten Patienten Steroide das Tuberkuloserisiko nicht erhöhen [13]. Keine Regel ohne Ausnahme: Eine Chemoprävention werde auch ich durchführen bei Patienten mit Asthma bronchiale, wenn das Erkrankungsrisiko außergewöhnlich hoch ist, z. B. bei einer Tuberkuloseanamnese unter 1 Jahr, bei Kindern mit zusätzlicher Immunsuppression z.B. durch zytostatische Behandlung sowie bei Patienten mit stark positivem Test (Tuberkulintest bei niedriger Konzentration) bzw. kürzlicher Tuberkulinkonversion.

\section{Literatur}

${ }^{1}$ American Thoracic Society. Treatment of Tuberculosis and Tuberculosis infection in Adults and Children. Am J Respir Crit Care Med 1994; 149: 1359-1374

${ }^{2}$ Haanaes DC, Bergmann A. Tuberculosis emerging in patients treated with corticosteroids. Eur J Respir Dis 1983; 64: 294-297

${ }^{3}$ Dörner AA et al. The development of tuberculous meningitis following cortisone therapy. Am Rev Tuberc 1951; 64: 564- 571

${ }^{4}$ King $\mathrm{E}$ et al. Tuberculosis following cortisone therapy. JAMA 1951; 146: $238-241$

${ }^{5}$ Kleinschmidt RF, Johnston JM. Miliary tuberculosis in a cortisone-treated patient: Case report with autopsy. Ann Intern Med 1951; 35: $694-702$

${ }^{6}$ Tegner W. Hazards of steroid treatment. Brit Med J 1962; 1 : $1587-1591$

${ }^{7}$ Kropp R. Probleme der prophylaktischen Gabe von Isonicotinsäurehydrazid bei langfristiger Corticosteroidtherapie. Med Klin 1969; 64: 2183-2196

${ }^{8}$ Konietzko N, Loddenkemper R. (Hrsg.). Tuberkulose. Stuttgart: Thieme Verlag Stuttgart, 1999

${ }^{9}$ American Thoracic Society. Targeted Tuberculin Testing and Treatment of Latent Tuberculosis infection. Am J Respir Crit Care Med 2000; 101: $221-247$

${ }^{10}$ Hernandez-Cruz B et al. Tuberculosis prophylaxis in patients with steroid treatment and systemic rheumatic diseases. Clin Exp Rheumatol 1999; 17(1): $81-87$

${ }^{11}$ Senderovitz T, Viskum K. Corticosteroids and Tuberculosis. Respiratory Medicine 1994; 88: 561 - 565
12 Bartmann K. Chemoprophylaxe und präventive Chemotherapie der Tuberkulose. Internist 1973; 14: 111 - 117

${ }^{13}$ Martos A et al. Steroids do not enhance the risk of development tuberculosis or other AIDS-related diseases in HIV-infected patients treated for pneumocystis carinii pneumonia. AIDS 1995; 9: 1037-1041

Prof. Dr. D. Kirsten

Krankenhaus Großhansdorf

Zentrum für Pneumologie und Thoraxchirurgie

Wöhrendamm 80

22927 Großhansdorf 